\title{
Subject-based Regional Anaesthesia Simulator combining Image Processing and Virtual Reality
}

\author{
Sebastian Ullrich ${ }^{1}$, Benedikt Fischer ${ }^{2}$, Alexandre Ntouba ${ }^{3}$, Jakob T. Valvoda ${ }^{1}$, \\ Andreas Prescher ${ }^{4}$, Torsten Kuhlen ${ }^{1}$, Thomas M. Deserno ${ }^{2}$, Rolf Rossaint ${ }^{3}$ \\ ${ }^{1}$ Virtual Reality Group, RWTH Aachen University \\ ${ }^{2}$ Institute of Medical Informatics, University Hospital Aachen \\ ${ }^{3}$ Clinic of Anaesthesia, University Hospital Aachen \\ ${ }^{4}$ Institute for Neuroanatomy, University Hospital Aachen \\ Email: s.ullrich@rz.rwth-aachen.de
}

\begin{abstract}
In this paper, a novel virtual reality-based simulator for regional anaesthesia is presented. Individual datasets of patients with nerve cords are created from medical scans with the help of advanced segmentation and registration algorithms. Techniques for interaction and immersive visualization are utilized by the simulator to improve training of medical residents.
\end{abstract}

\section{Introduction}

Regional anaesthesia encompasses several techniques for blocking the nerve supply to specific parts of the human body. Common techniques for upper and lower extremities are the axillary brachial plexus block and the femoral nerve block, respectively. After positioning the patient, the needle insertion site is determined with the help of surface and anatomic landmarks. An electric nerve stimulator is connected to the needle used for the procedure in order to locate nerve cords with muscular response (e.g., twitches in the hand or knee). After successful localization, local anesthetic is injected to block the desired nerve.

One essential requirement for simulation is a precise, anatomic plausible model of the nerve cords. In addition, representations of bones, blood vessels, musculature and skin tissue are needed to frame the peripheral nerve system. Current technology of medical image acquisition does not capture nerve tissue sufficiently. Therefore, advanced segmentation algorithms must be researched. The simulation itself should allow to train all steps of a typical procedure as described above. Currently, there is no extendable, software-based simulator for regional anaesthesia. Even though specialized haptics solutions are used by the applications described in the following section, clinical acceptance is very low. Reasons are restrictions to single datasets, rigid virtual patients that can not be repositioned, no proper training of needle insertion site localization and lack of simulators with support for peripheral block. On this account, training is still done on living patients. However, because errors in anaesthesiology can be lethal, there is an urgent necessity to find alternatives for training in medical education. 
Fig. 1. Overview of the system

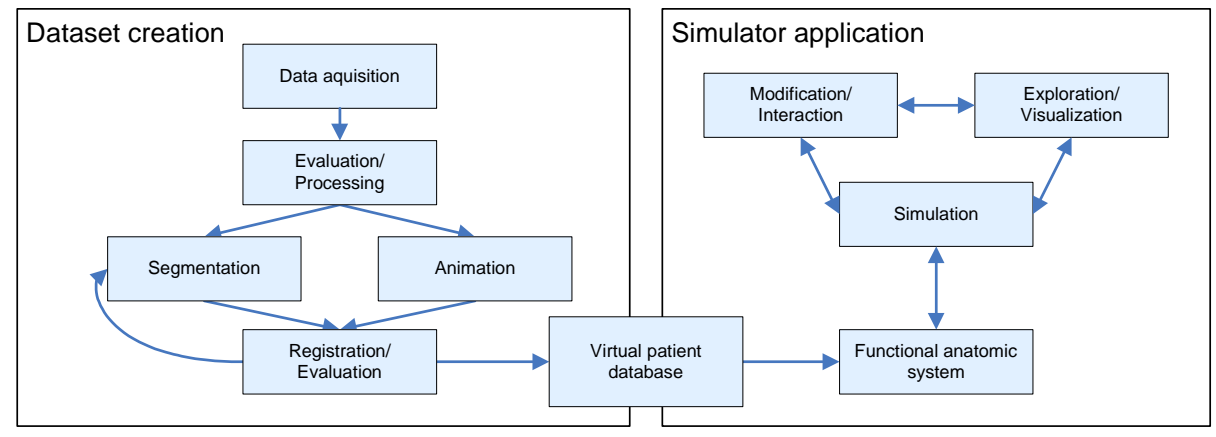

\section{State of the art and motivation}

The related work comprises segmentation and registration for dataset creation and simulation approaches.

The creation of appropriate datasets requires segmentation and registration of volumetric data from medical diagnostic imaging. Segmentation approaches for volumetric data can be divided into voxel-, surface-, and region-based as well as hybrid variants [1]. Standard registration approaches include point-to-point correspondence [2], extensions of the iterative closest point method for elastic registration [3], thin plate splines [4], boundary mapping [5], computational fluid dynamics [6], as well as daemon-based registration [7], which have been used already for mappings between an atlas and patient data [8].

A comprehensive overview of medical simulators is given in [9]. We have focused our research on topics related to regional anaesthesia. There are many needle simulators for different areas of applications, e.g., acupuncture [10], lumbar puncture $[11,12]$ and intravenous procedures [13]. Even though there are examples for epidural blocking procedures [14, 15], these approaches are based on static datasets (i.e., due to a rigid vertebral column the virtual patient can not bend forward) and are not adaptable to peripheral blocks of limbs.

\section{Methods}

The entire data flow is visualized in Figure 1. The two major parts, dataset creation and simulator application are linked via a virtual patient database.

\subsection{Dataset creation and processing}

Data acquisition and processing. The primary goal of data acquisition is to collect suitable/feasible data for segmentation of peripheral nerve cords. Therefore, initial efforts are focused on experimenting with parameters and time constants involved in relaxation processes of the tissue nuclei to improve contrast of 
magnet resonance imaging (MRI). In order to create a representative database, medical imaging is being conducted on different constitutional types.

Segmentation. A hybrid segmentation approach is applied. While structures such as skin, bones and musculature are clearly separated in the MRI scans, the nerve cords are not visualized with sufficient contrast to support an automatic segmentation. Therefore, a knowledge-based approach is used, where the relative position of the nerve cords and the arteries is defined in order to guide the segmentation process. In particular, the structural prototypes that have been defined in [16] are extended to the three-dimensional data source.

Registration. The registration has to match atlas-based information to the contents of the patient-specific images. At the current state, a daemon-based approach is favored, which has been successfully applied for similar purposes [8].

Animation. Typical muscular responses of the limbs induced by electric nerve stimulation are captured with optical tracking of markers attached to sections of the extremities. Additionally, the movement of single digits is captured with a data glove. The raw data of both approaches is used to compute the joint movements and store it as an animation sequence. With retargeting approaches, the data can be applied to all constitutional types and must be recorded only once.

\subsection{Simulator application}

The simulator application is being developed for virtual environments and consists of several modules (Fig. 1). The work is based on an extensible architecture that combines data, simulation, visualization and interaction [17].

Functional anatomy system. Data separation is a fundamental aspect of the proposed architecture. We conceived a structured approach that resembles the human organism as defined by the systematic and functional anatomy. To share common data and to create interlinks between algorithms, abstract control entities are designed that emulate the basic setup of physiological systems. The model-view-controller pattern is utilized to establish a separation of algorithms and data.

Simulation. The simulation algorithms operate on the data provided by the functional anatomy system. An interactively induced change of the pose of a virtual patient (for a procedure) is resolved by kinematic solutions and moves anatomical structures that are attached to each other accordingly. For nerve impulse transmission, a tree structure of nerve cords is implemented. This network is also used for visualization, collision detection and animation. Finite elementbased deformation algorithms are being adapted for needle penetration. 
Fig. 2. MRI slice with N. femoralis (a), prototype of the simulator on a Desktop-VR system (b) and interactive changes in posture of a virtual patient (c)

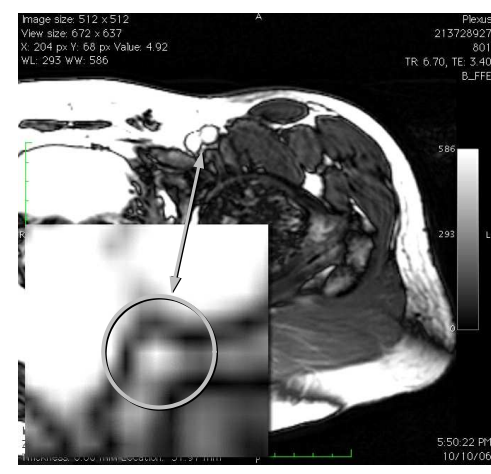

(a)

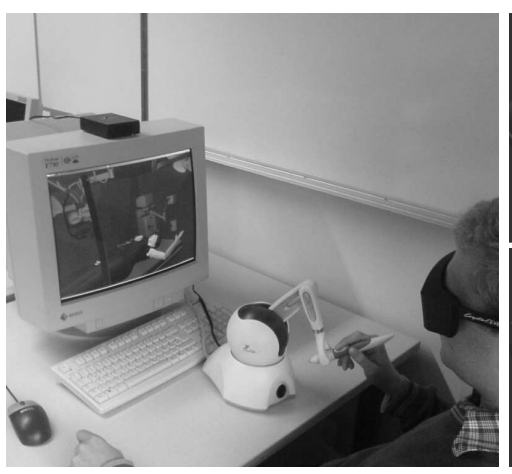

(b)

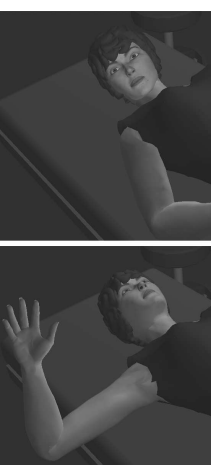

(c)

Visualization. Visualization is needed to enable an explorative analysis and to interactively render realistic geometric representations of the virtual patient and the instruments. We use vertex blending techniques to render the deformable skin surface. For evaluation purposes, the skin can be turned transparent to reveal anatomic visualizations of virtual bones, musculature, vessels and nerves.

Interaction. Besides anatomic realism, one of the most important requirements is an intuitive interaction interface. Therefore, the interaction algorithms support different input devices and allow to record a session during training for later evaluation. Customized haptic input devices are used for palpation to localize the needle insertion site realistically and are also used for needle operation.

\section{Results}

Several series of medical imaging scans have been evaluated. In comparison, MRI scans have yielded the best results (Fig. 2a), which can be explained by the anatomical soft tissue properties of nerve filaments. Segmentation algorithms are being adapted.

The architecture for the simulation of a virtual patient has been implemented. Virtual musculature and nerve cords are already simulated and can also be visualized. The application can be used in various virtual environments, varying from Desktop-VR to large projection environments (Fig. 2b). Several algorithms for interaction techniques are being adapted (Fig. 2c).

\section{Discussion and conclusion}

Datasets containing nerve cords are created with the help of hybrid segmentation algorithms that are in addition knowledge-based guided. The system renders an 
anatomical patient specific model in virtual environments that can be explored, intuitively controlled and manipulated. Additionally, evaluations are conducted by medical experts on a regular base to insure authentic data sets and plausible simulation. The final goal is to use the developed software as a new educational device for training of regional anaesthesia procedures.

\section{Acknowledgments}

This work was funded by the START-Programm (UK Aachen) and also supported by a grant from the German Research Foundation (DFG, KU 1132, LE 1108, RO 2000).

\section{References}

1. Lehmann TM, Hiltner J, Handels H. Handbuch der Medizinischen Informatik. 2nd ed. Carl Hanser Verlag; 2005. 361-424.

2. Moshfeghi M. Elastic matching of multimodality medical images. CVGIP: Graphical Models and Image Processing 1991; 271-282.

3. Rangarajan A, Chui H, Mjolsness E, et al. A robust point matching algorithm for autoradiograph alignment. Medical Image Analysis 1997;4(1):379-398.

4. Barret HH, Gmitro AF, editors. Information Processing in Medical Imaging. Springer; 1991. 326-342.

5. Davatzikos CA, Prince JL, Bryan RN. Image registration based on boundary mapping. IEEE Trans Med Imaging 1996;15(1):112-115.

6. Christensen GE. Deformable shape models for anatomy. Ph.D. thesis. Sever Institute of Technology, Washington University, St. Louis, MO, USA; 1994.

7. Thirion JP. Image matching as a diffusion process: An analogy with Maxwell's demons. Medical Image Analysis 1998;2(3):243-260.

8. Handels H, Horsch A, Lehmann TM, Meinzer HP. Bildverarbeitung für die Medizin. Springer; 2001.

9. Liu A, Tendick F, Cleary K, Kaufmann C. A survey of surgical simulation: Applications, technology, and education. Presence 2003;12(6):599-614.

10. Leung KM, Heng PA, Sun H, Wong TT. A haptic needle manipulation simulator for chinese acupuncture. In: Procs MMVR; 2003. 187-189.

11. Gorman P, Krummel T, Webster R, Smith M, Hutchens D. A prototype haptic lumbar puncture simulator. In: Procs MMVR; 2000. 106-109.

12. Färber M, Heller J, Handels H. Virtual reality simulator for the training of lumbar punctures. In: Procs CURAC; 2006. 126-127.

13. Rawn CL, Reznek MA, et al. Validation of an IV insertion simulator: Establishing a standard simulator evaluation protocol. In: Procs MMVR; 2002.

14. Blezek DJ, Robb RA, Martin DP. Virtual reality simulation of regional anaesthesia for training of residents. In: HICSS; 2000. 5022-5029.

15. Glassenberg R. Virtual epidural. In: Procs IMMS; 2004.

16. Fischer B, Winkler B, Thies C, Güld MO, Lehmann TM. Strukturprototypen zur Modellierung medizinischer Bildinhalte. Procs BVM 2006; 71-75.

17. Valvoda JT, Kuhlen T, Bischof CH. Interactive virtual humanoids for virtual environments. In: Eurographics Symposium on Virtual Environments; 2006. 9-12. 